\title{
BMJ Open Meta Salud Diabetes study protocol: a cluster-randomised trial to reduce cardiovascular risk among a diabetic population of Mexico
}

\author{
Samantha Sabo, ${ }^{1}$ Catalina Denman Champion, ${ }^{2}$ Melanie L Bell, ${ }^{3}$ \\ Elsa Cornejo Vucovich, ${ }^{2}$ Maia Ingram, ${ }^{1}$ Celina Valenica, ${ }^{4}$ \\ Maria del Carmen Castro Vasquez, ${ }^{2}$ Eduardo Gonzalez-Fagoaga, ${ }^{4}$ \\ Jill Geurnsey de Zapien, ${ }^{1}$ Cecilia B Rosales ${ }^{4}$
}

To cite: Sabo S, Denman Champion C, Bell ML, et al. Meta Salud Diabetes study protocol: a cluster-randomised trial to reduce cardiovascular risk among a diabetic population of Mexico. BMJ Open 2018;8:e020762. doi:10.1136/ bmjopen-2017-020762

- Prepublication history for this paper is available online. To view these files, please visit the journal online (http://dx.doi. org/10.1136/bmjopen-2017020762).

\section{SS, CDC, MLB and CBR} contributed equally.

Received 30 November 2017 Accepted 12 December 2017

\section{Check for updates}

${ }^{1}$ Department of Health Promotion Sciences, Mel \& Enid Zuckerman College of Public Health University of Arizona, Tucson, Arizona, USA

${ }^{2}$ Center for Health and Society Studies, El Colegio de Sonora, Hermosillo, Sonora, Mexico ${ }^{3}$ Department of Epidemiology and Biostatistics, Mel and Enid Zuckerman College of Public Health, University of Arizona, Tucson, Arizona, USA ${ }^{4}$ Division of Public Health Practice \& Translational Research, University of Arizona Mel and Enid Zuckerman College of Public Health, Phoenix, Arizona, USA

Correspondence to Dr Cecilia B Rosales; crosales@email.arizona.edu

\section{ABSTRACT}

Introduction Northern Mexico has among the highest rates of cardiovascular disease (CVD) and diabetes in the world. This research addresses core gaps in implementation science to develop, test and scale-up CVD risk-reduction interventions in diabetics through a national primary care health system.

Methods and analysis The Meta Salud Diabetes (MSD) research project is a parallel two-arm cluster-randomised clinical behavioural trial based in $22(n=22)$ health centres in Sonora, Mexico. MSD aims to evaluate the effectiveness of the MSD intervention for the secondary prevention of CVD risk factors among a diabetic population $(n=320)$ compared with the study control of usual care. The MSD intervention consists of 2-hour class sessions delivered over a 13-week period providing educational information to encourage sustainable behavioural change to prevent disease complications including the adoption of physical activity. MSD is delivered within the context of Mexico's national primary care health centre system by health professionals, including nurses, physicians and community health workers via existing social support groups for individuals diagnosed with chronic disease. Mixed models are used to estimate the effect of MSD by comparing cardiovascular risk, as measured by the Framingham Risk Score, between the trial arms. Secondary outcomes include hypertension, behavioural risk factors and psychosocial factors.

Ethics and dissemination This work is supported by the National Institutes of Health, National Heart Lung and Blood Institute (1R01HL125996-01) and approved by the University of Arizona Research Institutional Review Board (Protocol 1508040144) and the Research Bioethics Committee at the University of Sonora. The first Internal Review Board approval date was 31 August 2015 with five subsequent approved amendments. This article refers to protocol V.0.2, dated 30 January 2017. Results will be disseminated via peer-reviewed publication and presentation at international conferences and will be shared through meetings with health systems officials.

Trial registration number NCT0280469; Pre-results.
Strengths and limitations of this study

- Implementation research using a cluster-randomised trial design.

- Group-based intervention delivered within a national public health system.

- Contributes to evaluating organisational barriers and facilitators to implement and scale chronic disease interventions in low-resource and medium-resource contexts.

\section{INTRODUCTION}

\section{Background and rationale}

Cardiovascular disease (CVD) is the leading cause of death in Mexico, accounting for over one-third of all mortality. ${ }^{1}$ The growing threat of mortality from CVD and diabetes in Mexico is devastating, rising by $9.5 \%$ in 2010 alone. $^{2}$ CVD and diabetes are among the top two causes of death in Mexico. ${ }^{3}$ From 2005 to 2014, diabetes-attributable deaths increased by 26870 and cardiovascular-attributable deaths increased by $38564 .{ }^{4}$ Mexico's National Health Program 2007-2012 states that $33 \%$ of deaths among Mexican women and $26 \%$ of deaths among Mexican men are caused by these illnesses. ${ }^{5}$ CVD is the leading cause of early death among people with diabetes. ${ }^{6}$ Individuals with diabetes are two to six times more likely to die from CVD than non-diabetics. ${ }^{7}$ Obesity, high blood pressure, high cholesterol and smoking each contributes to high rates of CVD among diabetics. ${ }^{8}$ Secondary prevention of modifiable risk factors for CVD is of primary importance for lowering premature death rates in Mexico. ${ }^{9}$

Recognising the need to address modifiable risk factors for diabetes and CVD is a global priority for countries undergoing an epidemiological transition such as Mexico. ${ }^{10}$ 


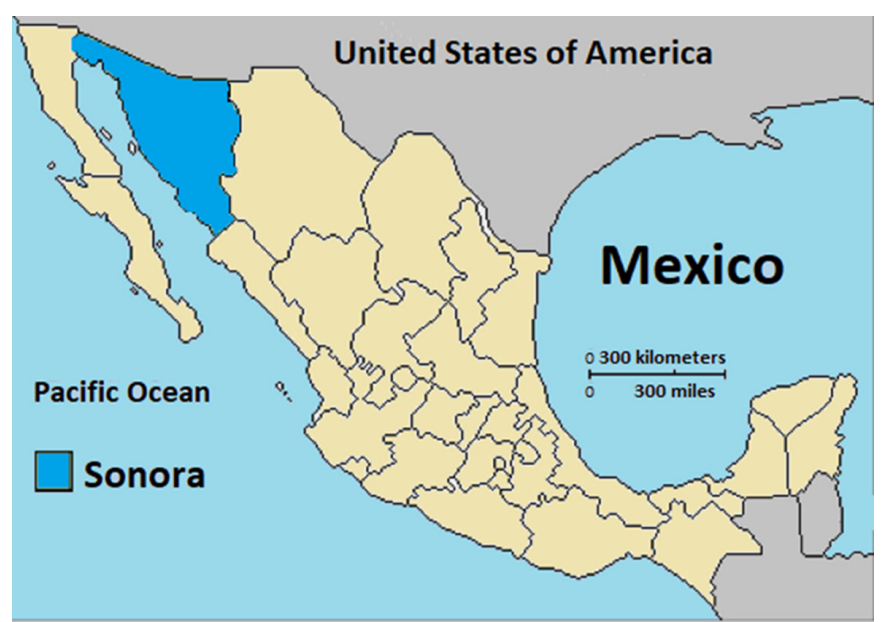

Figure 1 Map of study location.

A primary objective of $\mathrm{WHO}$ is to promote non-communicable disease interventions that address behavioural risk factors such as tobacco use, unhealthy diets and sedentary behaviour. ${ }^{11}$ The Mexican government has stated the need for adoption of scalable, evidence-based interventions that address healthy lifestyle promotion and disease management for patients with diabetes, their families and their communities, ${ }^{12}$ particularly given the fact a small percentage of the diabetic population in Mexico report successfully managing CVD-associated risk factors. ${ }^{13} \mathrm{An}$ average of $31.5 \%$ of Mexico's population have hypertension and $47.3 \%$ of individuals with hypertension are unaware of their condition. ${ }^{14}$ Thirty-two per cent (32\%) of the population in Mexico was categorised as obese in 2012 with body mass index (BMI) alone. ${ }^{15}$ Using the waist circumference BMI measure, almost $83 \%$ of women and $65 \%$ of men have abdominal obesity. ${ }^{2}$ Mexico presents rich opportunities to address secondary prevention of CVD among the diabetic population via culturally and contextually appropriate, evidence-based interventions.

The Northern Mexico state of Sonora is located on the US-Mexico border and shares its northern border with the state of Arizona (figure 1). In the last decade, Sonora had the highest hypertension rate and fifth highest abdominal obesity prevalence in Mexico, according to the country's National Health and Nutrition Survey (NHANES), Encuesta Nacional de Salud y Nutrición (ENSANUT). ${ }^{15} 16$ ENSANUT 2012 data indicate that Sonora has a 78.3\% prevalence of abdominal obesity, exceeding the national rate of $74.0 \% .^{15}$ The high prevalence rates for diabetes-associated and CVD-associated risk factors places Sonora at a disproportionately high disease risk burden that exceeds the disease risk burden of most other states in Mexico.

\section{Objectives}

The Meta Salud Diabetes (MSD) curriculum was designed by El Colegio de Sonora and the University of Arizona as part of 'Tools and practices to reduce CVD and complications in the diabetic population in Mexico', a project funded by the National Institutes of Health
(NIH) National Heart, Lung and Blood Institute as part of the Global Alliance for Chronic Diseases. The MSD behavioural intervention aims to reduce clinical risks for developing CVD among diabetic populations and is implemented in collaboration with the Sonora Ministry of Health. The research study's two primary objectives are: (1) to test the effectiveness of a CVD secondary prevention intervention among a diabetic population and (2) engage in a study that assesses the implementation of the MSD intervention to identify the strengths and limitations faced by each study affiliated clinical site.

\section{Trial design}

This is a parallel two-arm cluster-randomised clinical behavioural trial based in $22(n=22)$ health centres in Sonora, Mexico (figure 2).

\section{METHODS: PARTICIPANTS, INTERVENTIONS AND OUTCOMES Study setting}

Mexico's centralised health system includes health coverage programmes that provide access to care to most adults via a national network of hospitals and health centres. ${ }^{17}$ State and nationally run hospitals and health centres are the usual source of care for most Mexicans. ${ }^{18} 19$ The centralised nature of the health system provides the context for the MSD intervention. In Sonora, Mexico, the vast majority of adults (82\%), are covered by national programmes, approximately $26 \%$ are specifically covered by the national health insurance programme-Seguro Popular-implemented in $2004(25.6 \%){ }^{20}$ Seguro Popular aims to provide access to care for families, many of which are low income, who had been excluded from social health insurance in the past. ${ }^{17}$

Under Seguro Popular, the Mexican Ministry of Health's Community Health Centers (Centros de Salud) employ registered nurses (RNs) and community health workers (CHWs), whose activities include maintaining a support group for people with diabetes. ${ }^{21}$ These self-help groups, known as Grupos de Ayuda Mutua (GAMs) are one of the strategies for secondary prevention in patients with diabetes established by the Mexican Ministry of Health diabetes programme. ${ }^{21}$ The most recent report estimates 8525 GAMs operating across Mexico with over 222270 patient participants throughout the country. ${ }^{22}$ National standards and operating procedures are outlined by the Ministry of Health to encourage a standardised delivery of care and outcomes to facilitate national GAM evaluation. ${ }^{21}$ Monitoring of the GAMs occurs at the jurisdictional level and those meeting the standards are certified. ${ }^{21}$ Despite governmental protocol and GAM certification, high variability remains across GAMs in group size (which averages approximately 26 individuals), sociodemographics and disease profile of the participants. Each GAM determines meeting frequency, location, as well as the chronic disease self-management, education and skill building offered. ${ }^{23}$ Variability in the 


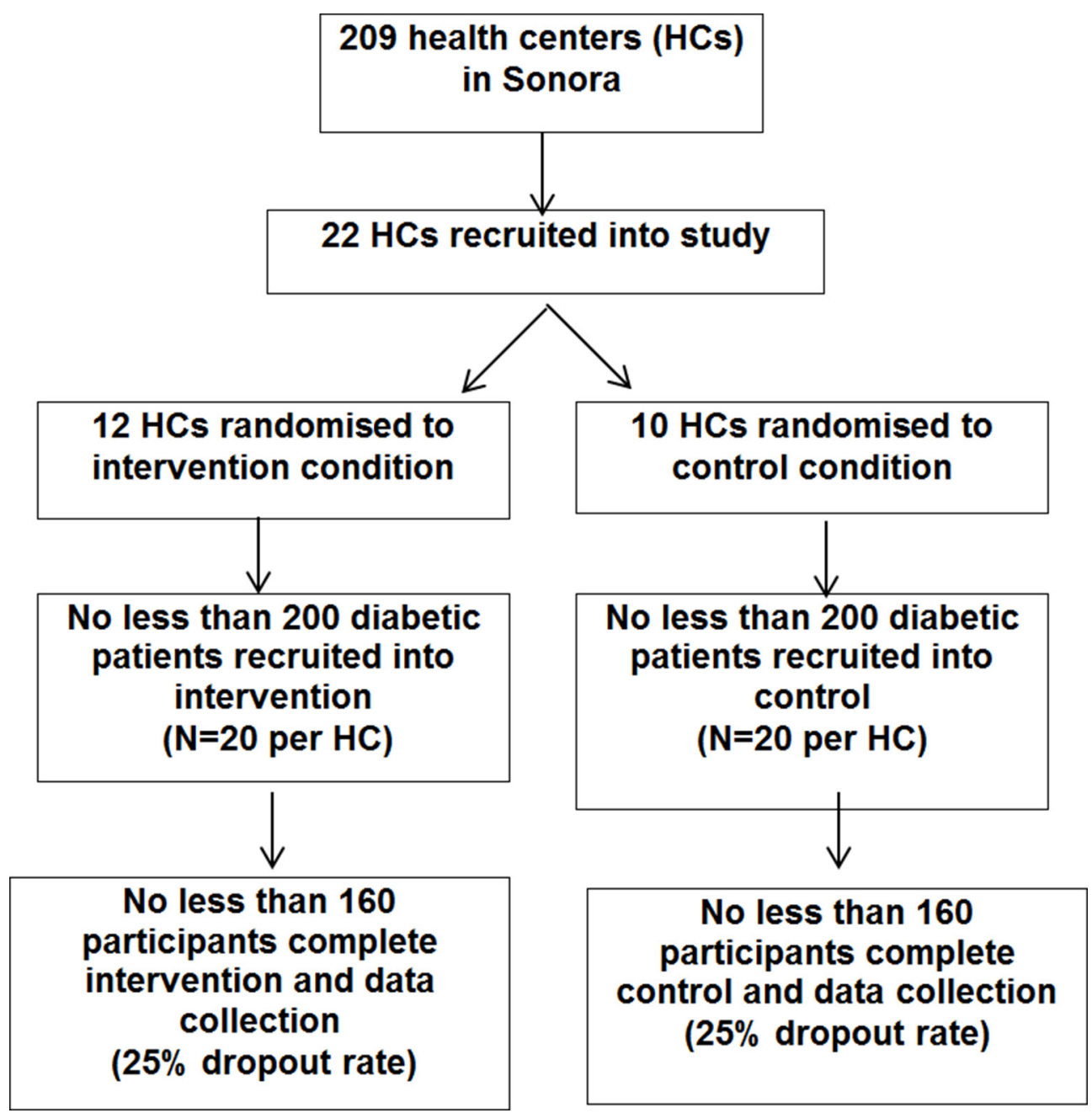

Figure 2 Flow chart of participation recruitment into cluster-randomised trial.

GAMs is often due to lack of adequate space, training and educational resources for the GAM coordinators to adequately support patient participants. Despite these challenges, the nationwide infrastructure of the GAMs, embedded within the national health centre system, represents an opportunity for a scale-up of an evidence-based intervention for CVD risk management among diabetics, as many Mexicans access healthcare at these health centres.

\section{Eligibility criteria}

Health centre eligibility

The health centres $(n=22)$ eligible for study inclusion are selected from the 209 Ministry of Health centres located in Sonora, Mexico. Health centres with the largest patient population are selected for study inclusion. Following identification of eligible health centres, the clinical sites are randomised to determine which health centres would be included in the intervention and control arms of the clinical trial.

\section{Participant eligibility}

Patients participating in an established GAM within a study clinical site are screened for study eligibility.
Study inclusion criteria for individual participants include: (1) adult age (18 years of age or older); (2) a medical provider diagnosis of diabetes and (3) be an established patient at the participating clinical site. Established patient is defined by this study as an individual who receives his/her primary care via the study clinical site (table 1).

Table 1 Study inclusion and exclusion criteria

\begin{tabular}{ll}
\hline Inclusion criteria & Exclusion criteria \\
\hline $\begin{array}{l}\text { Aged at least } \\
\text { 18years }\end{array}$ & Aged less than 18years \\
$\begin{array}{l}\text { Diagnosed with } \\
\text { type 2 diabetes }\end{array}$ & Not diagnosed with type 2 diabetes \\
$\begin{array}{l}\text { Give written } \\
\text { informed consent }\end{array}$ & $\begin{array}{l}\text { Pre-existing significant comorbid } \\
\text { condition precluding participation in } \\
\text { physical activity, as determined by } \\
\text { physical activity risk assessment and } \\
\text { medical provider }\end{array}$ \\
\hline
\end{tabular}

All research is conducted in the country of Mexico; therefore, it is assumed that all participants are Mexican nationals and speak Spanish as their primary language. 


\section{Intervention}

This section is organised by the Template for Intervention Description and Replication) checklist. ${ }^{24}$

The MSD curriculum is an individual-level, behaviouralbased intervention that provides nutritional education, social support and encourages physical activity as secondary prevention of CVD among diabetic populations in Mexico. The intervention builds on the MSD curriculum, a primary prevention educational intervention adapted from an evidence-based programme titled Pasos Adelante. ${ }^{25} 26$ Pasos Adelante is a chronic disease prevention curriculum provided in Spanish with Mexican nationals or first generation Mexican immigrants living in US-Mexico border communities and recognised by the Centers for Disease Control and Prevention (CDC) as a 'Winnable Battle' intervention (http://bit.ly/ $2 \mathrm{mLKJaD}){ }^{26}$ The primary prevention curriculum known as Meta Salud has demonstrated efficacy in lowering physiological risk factors associated with diabetes and CVD in Sonora. ${ }^{25-27}$

\section{MSD curriculum}

The MSD intervention consists of 2-hour class sessions delivered over a 13-week period (table 2). The MSD curriculum focuses on providing educational information to encourage sustainable behavioural change to prevent disease complications including the adaptation of physical activity. The curriculum places an emphasis on the importance of nutrition and physical activity. MSD components include: (1) educational curriculum delivered in a support group context that encourages individuals with diabetes to make sustainable behavioural changes to better manage their chronic illness, prevent complications and prevent CVD. The curriculum is culturally relevant, context-specific and tailored to meet the needs of the study population; (2) the intervention is couched in a participatory methodology to promote a community of learning with the goal of providing a supportive space for people at risk for developing CVDs and (3) physical activity is a strategic element of the intervention aimed to increase exercise and build supporting social networks within the intervention to help prevent and manage chronic diseases. Physical activity is embedded within each session to foster long-term maintenance of health behaviours and positive emotional well-being.

MSD intervention design consists of four stages. These four stages involve a transdisciplinary team of public health experts in nutrition, chronic disease management and psychology. ${ }^{25}$ These public health experts remain involved with the study implementation to ensure the MSD intervention meets its delineated objectives within the study population. Stage 1 consisted of the revision of protocols, programmes, and procedures that are specific to meeting the needs of diabetic populations seeking to curtail the development of CVD. ${ }^{25}$ Stage 2 consists of fieldwork to assess the participating health centre sites, the GAMs and the patient populations of each respective health centre. ${ }^{25}$ Stage 3 includes a rigorous literature review to capture the current science on behavioural and lifestyle interventions for nutrition, physical activity, stress management and mental health associated to diabetes, CVD and general chronic disease. ${ }^{25}$ Stage 4 is the final adaptation of intervention materials to integrate diabetes management education. ${ }^{25} 28$

MSD is guided by two primary behavioural change theories, the Trans Theoretical Model of Behaviour Change and Social Cognitive Theory. ${ }^{29} 30$ These two behavioural change theories assume, respectively, that behaviour modification is a multistage process in which people move through stages of readiness for change, and that they do so in the context of reciprocal relationships with their environment, behaviour and cognition. ${ }^{29} 30$ MSD is guided by specific adult learning theories, including meaningful learning theory and popular education. ${ }^{31} 32$ Both approaches acknowledge the agency of adult learners to integrate knew knowledge into what is already known and create a cognitive structure that makes sense of their own surroundings and situations. ${ }^{3132}$ Through these approaches, MSD privileges the coconstruction of knowledge among participants and assumes MSD facilitators and participants are colearners. Through meaningful learning theory and popular education, MSD encourages critical thinking about empowerment, personal and collective agency and particularly their right to quality healthcare services. Furthermore, the overarching framework for MSD is provided by salutogenesis, an asset-based model that searches for the origins of health (as opposed to the origins of disease sought by pathogenesis) and specifically addresses the role of stress and what Antonovsky has termed a sense of coherence. ${ }^{33}$ Finally, because of the implementation science focus of our trial, we also draw on the socialecological model, which supports our ability to assess, systematically, the potential for scale-up of MSD from the interpersonal, organisational, community and systems levels. $^{34}$

A health professional within a regularly scheduled GAM meeting delivers MSD. GAMs are facilitated by a variety of health professionals, including a RNs, CHWs, MDs and in some clinical sites an interdisciplinary team of health centre staff, including interns, facilitate GAM meetings. Health professionals who deliver the MSD curriculum receive a 2-day, face-to-face training session in a group setting. Training sessions use didactic and interactive popular education techniques to both demonstrate and provide opportunities for practice and role-play for each of MSD's 13 sessions.

Fidelity is documented by MSD research staff observation of most of the 13 sessions at all intervention sites. Fidelity tracks the extent to which the goals of each session are met, instructions for each activity are followed, as well evaluates the level of quality and consistency in facilitation across the intervention sites. The observers are trained in observation techniques that allow them to document the activities of the session, 
Table 2 Meta Salud Diabetes (MSD) knowledge, attitude and behaviour support sessions

\begin{tabular}{cl}
\hline Session & Session title \\
\hline 1 & Introduction to MSD \\
2 & $\begin{array}{l}\text { Living a healthy life with } \\
\text { diabetes }\end{array}$ \\
\end{tabular}

\section{Session learning goals}

By the end of this session participants will have:

Learnt the objectives of the MSD programme.

- Shared expectations about participation in the programme.

- Developed group agreements on coexistence and cohesion.

- Generated a collective vision of 'health'.

- Reflected on their current health status to identify strategies and goal setting for healthier habits to manage diabetes and cardiovascular disease (CVD). diabetes

- Learnt more about diabetes and how it works in the body.

- Identified symptoms and consequences of diabetes that you should discuss with your doctor.

- Learnt complications of diabetes and its relation to heart disease, kidneys, eyes and feet, as well as tips to prevent and reduce.

- Identified self-management techniques to live a health life with diabetes.

Are you at risk of developing
CVD?
Maintaining a healthy weight
Lentified the relationship between CVD and diabetes.
Learnt symptoms of a heart attack and how symptoms differ between men and women.
Learnt maintaining a healthy weight and BMI is helpful for diabetes control.
Ligh blood pressure and CVDs.
Lnderstands that fad diets and 'miracle' products are not effective and may be harmful to health.
Learnt and used 'My Healthy Plate' to create a healthier diet.
Learnt how gradual changes contribute to habits towards healthy weight loss and maintenance.

5 Benefits of physical activity Learnt physical activity helps achieve effective diabetes control, prevent CVD and improve overall health.

Learnt adult daily recommendations for physically activity $(15 \mathrm{~min})$ and the importance of increasing and incorporating physical activity into daily activities.

Learnt health benefits of frequent physical activity.

- Learnt a brisk walk is an easy activity that almost everyone can perform taking into account their physical characteristics.

$6 \quad$ Glucose and sugar

- Understood how blood glucose works in the body.

- Learnt appropriate levels of blood glucose for diagnosed diabetics.

- Differentiated between hypoglycaemia and hyperglycaemia.

- Identified sugar content of common drinks.

- Learnt physical activity contributes to stable blood glucose.

\begin{tabular}{|c|c|c|}
\hline 7 & $\begin{array}{l}\text { Everything you need to } \\
\text { know about high blood } \\
\text { pressure, salt and sodium }\end{array}$ & $\begin{array}{l}\text { Learnt definitions of high blood pressure and how it is measured. } \\
\text { Learnt how to prevent hypertension and stroke. } \\
\text { Identified appropriate amount of sodium for the body and how allowable daily sodium intake } \\
\text { accumulates. }\end{array}$ \\
\hline 8 & $\begin{array}{l}\text { Control your cholesterol, eat } \\
\text { less fat }\end{array}$ & $\begin{array}{l}\text { Learnt what cholesterol is and how it affects the body. } \\
\text { Learnt recommended cholesterol and triglycerides levels. } \\
\text { Learnt the steps to take to control of cholesterol and triglycerides levels. } \\
\text { Learnt about different types of fat. } \\
\text { Learnt how to cook with less fat. }\end{array}$ \\
\hline 9 & Is our community healthy? & $\begin{array}{l}\text { Reflected on how our environment influences our ability to make healthy choices. } \\
\text { Identified aspects of the community which promote and detract from the adoption of healthy } \\
\text { habits. } \\
\text { Learnt how certain people and groups contribute to building healthy communities. } \\
\text { Learnt how to create and use a community improvement plan. }\end{array}$ \\
\hline 10 & $\begin{array}{l}\text { Enjoy life with emotional } \\
\text { well-being }\end{array}$ & $\begin{array}{l}\text { Identified the relationship between emotions and health. } \\
\text { Defined depression and its relationship to diabetes. } \\
\text { Reflected on personal emotional health and how it affects personal food choices, use of alcohol } \\
\text { and tobacco. } \\
\text { Listed strategies to promote emotional well-being. }\end{array}$ \\
\hline 11 & $\begin{array}{l}\text { Effective management } \\
\text { of diabetes is a shared } \\
\text { responsibility }\end{array}$ & $\begin{array}{l}\text { Learnt the importance of taking action to improve diabetes control and pharmacological and non- } \\
\text { pharmacological treatments. } \\
\text { Recognised barriers and strategies to adhere to treatment. } \\
\text { Raised awareness of the importance of collaborating with one's medical team to improve health. } \\
\text { Learnt to clarify needs and concerns during medical appointments. } \\
\text { Learnt one's rights as patients and practised implementing these rights with medical care team. } \\
\text { Developed strategies for a respectful relationship with medical personnel. }\end{array}$ \\
\hline
\end{tabular}




\begin{tabular}{|c|c|c|}
\hline Session & Session title & $\begin{array}{l}\text { Session learning goals } \\
\text { By the end of this session participants will have: }\end{array}$ \\
\hline 12 & $\begin{array}{l}\text { Enjoy healthy meals with } \\
\text { friends and family }\end{array}$ & $\begin{array}{l}\text { Reflected on healthy aspects of traditional cuisine that can be incorporated into current eating } \\
\text { habits. } \\
\text { Used My Healthy Plate to adapt what they have learnt about healthy eating into their daily lives. } \\
\text { Reviewed suggestions for healthy meals and reflected on how advertising affects buying habits of } \\
\text { food and health products. }\end{array}$ \\
\hline 13 & Review and graduation & $\begin{array}{l}\text { Reviewed main points of each session through interactive games. } \\
\text { Shared achievements and experiences during the programme. } \\
\text { Evaluated the programme and provided suggestions for improvement. } \\
\text { Wrote a letter to themselves to continue to work on reaching healthy goals. } \\
\text { Received recognition for participation in the programme. } \\
\text { Celebrated with participant group. }\end{array}$ \\
\hline
\end{tabular}

and take detailed notes on how the intervention is received and the quality of interactions between the facilitator and participants and between participants themselves. Following completion of the trial, two research staff will independently review the detailed notes of at least $20 \%$ of all the sessions observed. Using a prepared fidelity checklist, the observers will indicate if each objective was introduced and whether all of the information related to the material was covered and all planned activities were carried out in the session. The observers will use a five-point Likert scale to evaluate the quality of the curriculum delivery and evaluate the extent to which the facilitator presented the information in an engaging, consistent and clear manner. In addition, the checklist will rate the extent to which the facilitator engages in interactive activities based on adult learning theories. The checklist includes observations on whether the facilitator focuses on participant learning and encourages them to be interactive; shows compassion to the participants without focusing on personal problems of any one participant; encourages participants to give examples of situations in the curriculum and to ask questions related to their own experience.

\section{Outcomes}

\section{Primary outcome}

As a secondary prevention intervention to reduce CVD risk among diabetic populations in Northern Mexico, the study uses the Framingham Risk Score (FRS) as the primary outcome measure. ${ }^{35}$ FRS is a reliable estimate of an individual's likelihood to develop CVD over a 10-year period. ${ }^{35}$ FRS uses age, total cholesterol, high-density lipoprotein cholesterol, blood pressure, smoking and diabetes to assess CVD risk in the study population. ${ }^{35} 36$ Validation studies have established FRS as an excellent tool for predicting CVD events in the USA among diverse populations. ${ }^{37} 38$ FRS has not been validated in Latin American populations. ${ }^{39}$ This clinical trial provides an opportunity to generate data towards the validation of FRS in predicting CVD in Latin American populations (table 3).

\section{Secondary outcomes}

Secondary outcomes consider anthropometric, behavioural risk factors and psychosocial factors associated with CVD and diabetes. MSD research staff follow the WHO Steps Manual anthropometric data collection protocols (http://bit.ly/2z33SXw) and a self-report interview questionnaire to collect behavioural risk factors and psychosocial factors. Self-report collection instruments have been validated and extensively used among Mexican and Mexican American populations. For behavioural risk factors, the self-report measures draw from the Spanish versions of the NHANES, the National Health Interview Survey, Mexico's National Health and Nutrition Survey (ENSANUT), Centers for Disease Control Behavioural Risk Factor Survey and the WHO International Physical Activity Scale. ${ }^{40-43}$ Self-report measures for psychosocial risk factors for CVD and diabetes draw from Problem Areas in Diabetes Questionnaire, Cohen's perceived stress scale as well as overall quality of life measures. ${ }^{44-46}$

\section{METHODS: ASSIGNMENT OF INTERVENTION, DATA COLLECTION, MANAGEMENT AND ANALYSIS}

Data collection

Data are collected from participants at three time points: baseline, 3 months (postintervention)and 12 months (9 months postintervention). Anthropometric measures, clinical measures and self-report data are collected at the three-respective data collection time points (table 3).

\section{Sample size}

To detect a standardised effect size of 0.4 for the FRS between the intervention and control groups at 3 months with at least $80 \%$ power, we calculate a sample size of 480 $(\mathrm{n}=480)$ participants. This calculation assumes 12 clusters for intervention arm and 10 for control (clinical site $\mathrm{n}=22$ ) (inflated by two in intervention arm to account for cluster dropout), 20 participants per cluster on average (inflated from $16 \%$ to $25 \%$ to account for participant dropout) and an intracluster correlation (ICC) of $0.03 .{ }^{47}$ This is a conservative ICC estimate based on reviews of several studies with clustering where the median ICCs 
Table 3 Primary and secondary outcome measures by measurement instrument and timing

\begin{tabular}{|c|c|c|c|}
\hline & Outcomes & $\begin{array}{l}\text { Measurement protocol/ } \\
\text { Survey source }\end{array}$ & Timing \\
\hline Anthropometric & $\begin{array}{l}\text { Height, weight, BMI, waist and hip } \\
\text { measures }\end{array}$ & WHO STEPS surveillance & $\begin{array}{l}\text { Baseline, } 3 \text { and } \\
12 \text { months }\end{array}$ \\
\hline Clinical & $\begin{array}{l}\text { Glycated haemoglobin (Haemoglobin } \\
\text { A1c); total cholesterol, LDL and HDL; } \\
\text { triglycerides; systolic and diastolic blood } \\
\text { pressure }\end{array}$ & WHO STEPS surveillance & $\begin{array}{l}\text { Baseline, } 3 \text { and } \\
12 \text { months }\end{array}$ \\
\hline Quality of life & Mental health physical health & BRFSS, ENSANUT & $\begin{array}{l}\text { Baseline, } 3 \text { and } \\
12 \text { months }\end{array}$ \\
\hline $\begin{array}{l}\text { Diabetes attitudes, wishes } \\
\text { and needs }\end{array}$ & Diabetes attitudes, wishes and needs & $\begin{array}{l}\text { Problem Areas in Diabetes } \\
\text { Questionnaire (PAID) }\end{array}$ & $\begin{array}{l}\text { Baseline, } 3 \text { and } \\
12 \text { months }\end{array}$ \\
\hline Physical activity & $\begin{array}{l}\text { Moderate and vigorous physical activity; } \\
\text { sedentary behaviour; walking behaviours }\end{array}$ & $\begin{array}{l}\text { International Physical Activity } \\
\text { Scale/WHO }\end{array}$ & $\begin{array}{l}\text { Baseline, } 3 \text { and } \\
12 \text { months }\end{array}$ \\
\hline Stress & & Cohen Perceived Stress Scale & $\begin{array}{l}\text { Baseline, } 3 \text { and } \\
12 \text { months }\end{array}$ \\
\hline
\end{tabular}

Measurement Protocols and Survey Sources: WHO STEPS Manual http://bit.ly/2z33SXw; Mexican National Health and Nutrition Survey (ENSANUT) http://bit.ly/2jGhAN7; Centre for Disease Control Behavioural Risk Factor Survey (BRFS) http://bit.ly/2ll1Tuj; PAID http://bit. ly/2bMkGLu; Cohen Perceived Stress Scale http://bit.ly/2zQtTgh. Data are collected for all study participants even if they discontinue or deviate from intervention protocols.

BMI, body mass index; BRFSS, Behavioural Risk Factor Surveillance System; ENSANUT, Encuesta Nacional de Salud y Nutrición; GACD, Global Alliance for Chronic Diseases; HDL,high-density lipoprotein; LDL, low density lipoproteins.

were found to be $0.01-0.02 .71,72$. This sample size also gives more than $80 \%$ power to detect a difference of $20 \%$ hypertension rates between the groups, assuming rates of $60 \%$ and $40 \%$. If the ICC is smaller, or more health centres are recruited, smaller differences can be detected. The difference in arms for which we powered on is feasible based on an ongoing evaluation study of CVD risk among patients of resource-intensive health centres in Sonora (such as the Unidades de Especialidades Médicas or Medical Specialty Units). A standardised effect size of 0.4 is considered small to medium effect and is likely to be clinically meaningful. ${ }^{48}$

\section{Recruitment}

Prior to study recruitment, research staff meet with all clinical staff from the study's clinical site to explain all aspects of the clinical trial and to discuss optimal approaches for recruitment and retention. A research staff member is assigned to each clinic to ensure ongoing communication and clarification throughout the duration of the study. Clinical staff that express interest in assisting with patient referral and recruitment are provided internal review board (IRB)-approved recruitment flyers and informed that they will not participate in the process of informed consent or data collection. Clinical staff will also be instructed to only provide study information found on the IRB recruitment flyers without providing potential study participants additional information. At least two staff members from each clinic who participate in GAM facilitation attend training on the study intervention in order to implement the MSD curriculum and other health centre staff (including clinic administrators, physicians, nurses and CHWs) meet with research staff to be fully informed of the study logistics and goals.

Staff within the study clinical sites identify patients eligible for study inclusion screening. The health provider, clinic staff or clinic personnel inform the patient of the study and provide an IRB approved recruitment flyer. Study recruitment flyers are also displayed at participating clinical sites for recruitment. Interested individuals are directed to contact the study staff responsible at the clinical site. Study staff inform the individual of the time and location of the GAM meeting where the enrolment activities and informed consent take place.

Retention activities include: (1) reminder telephone calls and text messages to participants from research staff; (2) development of a phone chain or text message chain among participants to remind each other about upcoming classes and measurement dates; (3) in person contact with the enrolled participant by clinical and research staff during intervention sessions or routine medical appointments. For both of the two follow-up measures, study staff make three and (4) attempts to contact the participant through telephone calls and or home visits. After the third unsuccessful attempt to make contact with the 
participant, the study categorises the participant as lost to follow-up.

\section{Assignment of interventions}

The trial has 22 participating clinical sites across Sonora, Mexico, which serve as the unit of randomisation. Clinics were randomised in a 1:1 ratio using stratified block randomisation with a variable block size. Stratification was done by geographical category (Central, North, South) and the three data collection cycles. Randomisation was performed using ralloc module of the statistical software Stata by the study biostatistician who had no contact with the clinical sites or the study participants. Due to the cluster randomisation, there was no concealment of the allocation of the clinics to intervention or control. This is not a blinded study, both intervention and control sites as well as study staff are aware of the allocation to intervention or control.

\section{Data collection}

Eligible individuals are invited to attend the MSD enrolment session. During the enrolment session, each individual participates in the study's informed consent process. Eligible individuals must provide informed consent prior to study enrolment. Only enrolled participants will participate in data collection. The informed consent protocol is conducted with every study participant. A research staff member provides a hard copy of the consent to the individual seeking to enrol in the study. Only research study staff conducts enrolment and monitors study enrolment. After the individual has successfully completed the informed consent process as per the study protocol, the participant undergoes baseline data collection consisting of: cholesterol, haemoglobin A1c, triglycerides, blood pressure, height, weight, waist circumference, hip circumference, demographic information and self-report questionnaire (table 3$)$.

\section{Data management}

All data are stored in the REDCap electronic data management system, an encrypted database housed on a dedicated server located in the University of Arizona, College of Medicine. REDCap is developed specifically around Health Insurance Prortability and Accountability Act security guidelines and is approved and endorsed by the University of Arizona Privacy Office and IRB.

\section{Statistical methods}

This is a parallel two-arm cluster-randomised trial to evaluate the effectiveness of MSD for the secondary prevention of CVD risk factors among a diabetic population versus the study control of usual care. The unit of randomisation is the health centre $(n=22)$. The primary outcome is the FRS, which is be measured at baseline, 3 months (postintervention) and 12 months (9 months postintervention). Secondary outcomes include hypertension, behavioural risk factors and psychosocial factors, as detailed above.
Descriptive statistics for each cluster and study participants are computed by arm. Differences between the intervention and control arms at 3 and 12 months are estimated from mixed models (linear for continuous outcomes and generalised with a binomial link for binary outcomes such as hypertension). Mixed models are used for the analysis of the data as it is of a correlated nature. Repeated measures on participants and patients within clusters will yield valid results if the data have a pattern of missingness of either missing completely at random or at random. ${ }^{49}$ If the missing data rate for the primary outcome is higher than $20 \%(\geq 20 \%)$, we perform sensitivity analysis appropriate for cluster-randomised trials. ${ }^{50}$ Adjusted estimates will also be reported to account for potential selection bias and or imbalance of key covariates in individual participants within health centres. Analysis is based on intention-to-treat, with which mixed models are consistent. ${ }^{51}$ Appropriate regression methods are used to undertake correlative analyses, to explore factors associated with greater changes in the FRS and hypertension.

\section{METHODS: MONITORING \\ Data monitoring}

A data monitoring committee was not established for this trial given its low intensity.

\section{Harms}

The research site coordinator designated to each health centre monitors and spontaneously reports adverse events and other unintended effects. All issues are documented by the site coordinator in the REDCap database at both the participant identification level and a separate internal use electronic journal that is dedicated to documentation and response to adverse events and unintended effects. Study site coordinators debrief regularly with the study coordinator to manage and address all issues within the guidelines set forth by the NIH. ${ }^{52}$

\section{ETHICS AND DISSEMINATION \\ Protocol amendments}

This article refers to protocol V.0.2, dated 1 January 2017.

\section{Consent}

Each clinic has an assigned clinical site research coordinator. The informed consent process is overseen by the clinical site research coordinator and is responsible for overall enrolment of all subjects. Research staff members will be trained on the informed consent procedure and be responsible for enrolling individual participants. Each individual will provide informed consent prior to being enrolled in the study. Only enrolled participants are engaged in data collection. The outlined informed consent protocol is conducted with every study participant. A research staff member is provided a physical copy of the consent to the individual seeking to enrol in the study. The research staff 
member reads the consent form loudly and clearly to the individual. After reading the consent form for the individual, the research staff member summarises the contents of the consent form. Following the summary, the research staff member asks the individual if they understood what was read to them and the summary they received and if she/he has any questions about what they reviewed together. If the individual agrees with the content of the consent form and participation in the study, she/he will then be asked to register in the research study's database. The individual then enters his/her name into the study's database and provides a signature on the electronic consent form.

\section{Confidentiality}

The study documents are electronically stored on computers and tablets on encrypted databases on a secure server affiliated with the UA. The computers and tablets are kept in locked storage cabinets when they are not in use at COLSON and/or UA.

\section{Access to data}

Only the Co-PI's and Co-Investigators of the study have access to the final trial data set and final list of study sites, and disclosure of contractual agreements that limit such access for investigators.

\section{Dissemination}

On completion of the trial, we will engage major dissemination strategies, including; (1) peer-reviewed publications in targeted journals; (2) scholarly presentations at scientific conferences and public health governance meetings; (3) interactive web-based promotional and training materials and (4) strategic informational and planning meetings with key institutions and centres of the Ministry of Health at the local, state and federal level. We will also leverage our existing partnerships and networks to disseminate findings, including the GACD Research Network, CDC Prevention Research Centers and the US-Mexico Border Health Commission, a binational governance body representative of the USA and Mexican Ministries of Health. Publications and presentations will involve and strengthen early-stage and late-stage binational research team members' capacity to translate research finding into recommendations for reduction of CVD risk and complications of both Mexican nationals and transnational populations of the US-Mexico border region. Web-based dissemination of promotional and training materials will be critical. El Colegio de Sonora and University of Arizona College of Public Health investigators have extensive expertise and capacity to use the internet to promote and train health professionals, including CHWs, as evidenced by the web portal for the original, UnitedHealth Chronic Disease Initiative funded Meta Salud programme (http://sitios.colson. edu.mx/metasalud/) with links maintained by the University of Arizona Prevention Research Center website. The existing Meta Salud portal will be leveraged to disseminate research findings and models for sustainability of the MSD programme.

Acknowledgements We would like to acknowledge the Ministry of Health of Sonora, Mexico, for their commitment to participating in this study designed to improve the health of their patients with diabetes.

Contributors SS, CDC and MLB contributed to the conceptualisation of the research design and methods and lead the writing and final review of the research protocol. ECV, MI, MdCCV, JGdZ and RBC conceptualised the research design and methods design, contributed to writing and critical review of the paper. CV and EG contributed content expertise to the study protocol and critically reviewed the final submission. CDC and RBC serve as co-principal investigators and approved final manuscript for publication.

Funding This work is supported by the National Institutes of Health, National Heart Lung and Blood Institute (NHLBI) grant number 1R01HL125996-01.

Competing interests None declared.

Patient consent Obtained.

Ethics approval The Collaborative Institutional Training Initiative (CITI) for research serves to certify all research study staff on human subjects. The protocol was also approved by the Research Bioethics Committee at the University of Sonora. The University of Arizona Human Subjects Institutional Review Board has reviewed and approved the study and all affiliated activities.

Provenance and peer review Not commissioned; peer reviewed for ethical and funding approval prior to submission.

Open Access This is an Open Access article distributed in accordance with the Creative Commons Attribution Non Commercial (CC BY-NC 4.0) license, which permits others to distribute, remix, adapt, build upon this work non-commercially, and license their derivative works on different terms, provided the original work is properly cited and the use is non-commercial. See: http://creativecommons.org/ licenses/by-nc/4.0/

(C) Article author(s) (or their employer(s) unless otherwise stated in the text of the article) 2018. All rights reserved. No commercial use is permitted unless otherwise expressly granted.

\section{REFERENCES}

1. Sistema Nacional de Información en Salud. Principales causes de mortalidad general, 2008. 2008 http://sinais.salud.gob.mx/ mortalidad.

2. Gutiérrez J, Rivera-Dommarco J, Shamah-Levy T, et al. Encuesta Nacional de Salud y Nutricion2012: resultados nacionales. México: Instituto Nacional de Salud Pública, 2012.

3. Pan American Health Organization (PAHO). Health in the Americas: 2012 edition. México, Washington: Pan American Health Organization, 2012.

4. Secretaria de Salud. Boletín de información estadística 2014-2015. 2015. http://www.dgis.salud.gob.mx/descargas/pdf/Boletxn_ InformacixnEstadxstica_14_15.pdf

5. Secretaria de Salud. Programa nacional de salud 2007-2012. Mexico D.F, 2012.

6. Morrish NJ, Wang SL, Stevens LK, et al. Mortality and causes of death in the WHO multinational study of vascular disease in diabetes. Diabetologia 2001;44(Suppl 2):S14-21.

7. Fan W. Epidemiology in diabetes mellitus and cardiovascular disease. Cardiovasc Endocrinol 2017;6:8-16.

8. Lorber D. Importance of cardiovascular disease risk management in patients with type 2 diabetes mellitus. Diabetes Metab Syndr Obes 2014;7:169-83.

9. Kontis V, Mathers CD, Bonita R, et al. Regional contributions of six preventable risk factors to achieving the $25 \times 25$ non-communicable disease mortality reduction target: a modelling study. Lancet Glob Health 2015;3:e746-757.

10. Gómez-Dantés H, Fullman N, Lamadrid-Figueroa H, et al. Dissonant health transition in the states of Mexico, 1990-2013: a systematic analysis for the Global Burden of Disease Study 2013. Lancet 2016;388:2386-402.

11. World Health Organization (WHO). 2008-2013 action plan for the global strategy for the prevention and control of non-communicable diseases. Geneva: World Health Organization, 2008. 
12. Secretaria de Salud. Estrategia nacional para la prevención y el control del sobrepeso, la obesidad y la diabetes. Mexico D.F: Secretaria de Salud, 2013. (accessed 20 Sep 2017).

13. Barquera S, Campos-Nonato I, Aguilar-Salinas $\mathrm{C}$, et al. Diabetes in Mexico: cost and management of diabetes and its complications and challenges for health policy. Global Health 2013;9:3.

14. Campos-Nonato I, Hernández-Barrera L, Rojas-Martínez R, et al. Hipertensión arterial: prevalencia, diagnóstico oportuno, control y tendencias en adultos mexicanos. Salud Publica Mex 2013;55(Suppl 2):144-50.

15. Barquera S, Campos-Nonato I, Hernández-Barrera L, et al. Prevalencia de obesidad en adultos mexicanos, ENSANUT 2012. Salud Publica Mex 2013;55(Suppl 2):151-60.

16. Barquera S, Campos-Nonato I, Hernández-Barrera L, et al. Hypertension in Mexican adults: results from the national health and nutrition survey 2006. Salud Publica Mex 2010;52(Suppl 1):S63-71.

17. Frenk J, González-Pier E, Gómez-Dantés O, et al. Comprehensive reform to improve health system performance in Mexico. Lancet 2006;368:1524-34.

18. Knaul FM, González-Pier E, Gómez-Dantés O, et al. The quest for universal health coverage: achieving social protection for all in Mexico. Lancet 2012;380:1259-79.

19. Atun R, de Andrade LO, Almeida G, et al. Health-system reform and universal health coverage in Latin America. Lancet 2015;385:1230-47.

20. Instituto Nacional de Salud Pública (INSP). Encuesta Nacional de salud y nutrición 2012. resultados por entidad federativa. Morelos,México: Instituto Nacional de Salud Pública, 2012.

21. Secretaria de Salud. Estrategia grupos de ayuda mutua enfermedades crónicas, lineamientos de operación 2016. Mexico: Secretaria de Salud, 2016

22. Fernandez BG. Estrategia de grupos de ayuda mutua enfermedades cronicas en Mexico. 2010 https://www.scribd.com/document/ 102465261/Estrategia-de-Grupos-de-Ayuda-Mutua-EnfermedadesCronicas-en-Mexico (accessed 13 Nov 2017).

23. Esqueda AL, Calderón AA, Jiménez RA, et al. Grupo de ayuda muta: estrategia para el control de diabetes e hipertensión arterial. Arch Cardiol Mex 2004;74:330-6.

24. Hoffmann TC, Glasziou PP, Boutron I, et al. Better reporting of interventions: template for intervention description and replication (TIDieR) checklist and guide. BMJ 2014;348:91687.

25. Denman CA, Bell ML, Cornejo E, et al. Changes in health behaviors and self-rated health of participants in Meta Salud: a primary prevention intervention of NCD in Mexico. Glob Heart 2015;10:55-61.

26. Staten LK, Cutshaw CA, Davidson C, et al. Effectiveness of the Pasos Adelante chronic disease prevention and control program in a US-Mexico border community, 2005-2008. Prev Chronic Dis 2012;9:E08.

27. Staten LK, Scheu LL, Bronson D, et al. Pasos Adelante: the effectiveness of a community-based chronic disease prevention program. Prev Chronic Dis 2005;2:A18.

28. Denman CA, Rosales C, Cornejo E, et al. Evaluation of the community-based chronic disease prevention program Meta Salud in Northern Mexico, 2011-2012. Prev Chronic Dis 2014;11:E154.

29. Prochaska JO, Velicer WF. The transtheoretical model of health behavior change. Am J Health Promot 1997;12:38-48.

30. Bandura A. Social cognitive theory: an agentic perspective. Annu Rev Psychol 2001;52:1-26.

31. Ausubel DP. The acquisition and retention of knowledge: a cognitive view. The Netherlands: Springer, 2000
32. Taylor P, Fransman J. Learning and teaching participation: exploring the role of higher learning institutions as agents of development and social change. UK: IDS, 2004.

33. Antonovsky A. Health, stress and coping. San Francisco, CA: Jossey-Bass, 1979.

34. McLeroy KR, Bibeau D, Steckler A, et al. An ecological perspective on health promotion programs. Health Educ Q 1988;15:351-77.

35. Wilson PW, D'Agostino RB, Levy D, et al. Prediction of coronary heart disease using risk factor categories. Circulation 1998;97:1837-47.

36. Lloyd-Jones DM, Wilson PW, Larson MG, et al. Framingham risk score and prediction of lifetime risk for coronary heart disease. Am J Cardiol 2004;94:20-4.

37. D'Agostino RB, Grundy S, Sullivan LM, et al. CHD Risk Prediction Group. Validation of the Framingham coronary heart disease prediction scores: results of a multiple ethnic groups investigation. JAMA 2001;286:180-7.

38. D'Agostino RB, Vasan RS, Pencina MJ, et al. General cardiovascular risk profile for use in primary care: the Framingham heart study. Circulation 2008;117:743-53.

39. Schargrodsky H, Hernández-Hernández R, Champagne BM, et al. CARMELA: assessment of cardiovascular risk in seven Latin American cities. Am J Med 2008;121:58-65.

40. Viner RM, Haines MM, Taylor SJC, et al. Body mass, weight control behaviours, weight perception and emotional well being in a multiethnic sample of early adolescents. Int $J$ Obes 2006;30:1514-21.

41. Groesz LM, Levine MP, Murnen SK. The effect of experimental presentation of thin media images on body satisfaction: a metaanalytic review. Int J Eat Disord 2002;31:1-16.

42. van den Berg P, Thompson JK, Obremski-Brandon K, et al. The Tripartite Influence model of body image and eating disturbance: a covariance structure modeling investigation testing the mediational role of appearance comparison. J Psychosom Res 2002;53:1007-20.

43. WHO. Non-Communicable Disease, Global Physical Activity Surveillance. 2018. World Health Organization. http://www.who.int/ ncds/surveillance/steps/GPAQ/en/ (accessed 19 Jan 2018).

44. McGuire BE, Morrison TG, Hermanns N, et al. Short-form measures of diabetes-related emotional distress: the Problem Areas in Diabetes Scale (PAID)-5 and PAID-1. Diabetologia 2010;53:66-9.

45. Cohen S, Kamarck T, Mermelstein R. A global measure of perceived stress. J Health Soc Behav 1983;24:385-96.

46. Ramírez MT, Hernández RL. Factor structure of the Perceived Stress Scale (PSS) in a sample from Mexico. Span J Psychol 2007;10:199-206.

47. Bell ML, McKenzie JE. Designing psycho-oncology randomised trials and cluster randomised trials: variance components and intra-cluster correlation of commonly used psychosocial measures. Psychooncology 2013;22:1738-47.

48. Cohen J. Statistical power analysis for the behavioral sciences. Hillsdale, New Jersey: Lawrence Earlbaum Associates, 1998.

49. Bell ML, Fairclough DL. Practical and statistical issues in missing data for longitudinal patient-reported outcomes. Stat Methods Med Res 2014;23:440-59.

50. Fiero $\mathrm{MH}, \mathrm{Hsu} \mathrm{CH}$, Bell ML. A pattern-mixture model approach for handling missing continuous outcome data in longitudinal cluster randomized trials. Stat Med 2017;36:4094-105.

51. Molenberghs $\mathrm{G}$, Thijs $\mathrm{H}$, Jansen I, et al. Analyzing incomplete longitudinal clinical trial data. Biostatistics 2004;5:445-64.

52. National Institute of Health (NIH). NHLBI adverse event and unanticipated problem reporting policy. 2017 https://www.ncbi.nlm. nih.gov/pubmed/ (accessed 13 Nov 2017) 